Check for updates

Cite this: J. Mater. Chem. C, 2021 9, 15721

Received 7th September 2021, Accepted 8th October 2021

DOI: 10.1039/d1tc04253j

rsc.li/materials-c

\title{
2D material hybrid heterostructures: achievements and challenges towards high throughput fabrication
}

\author{
Laura Maggini (D)* and Rúben R. Ferreira (D)
}

\begin{abstract}
2D materials have attracted tremendous attention since the discovery of graphene, because of their unique optical/electronic/mechanical properties, and their manipulable bidimensional morphology. Since forthcoming technologies require a stringent yet faceted portfolio of features, hardly feasible using a single pristine material, the demand for property tuning and multifunctionality has led to the development of hybrid 2D material heterostructures to modulate and exploit the synergy between two or more materials and achieve novel properties. Because of their straightforwardness in implementation and rich variety of possible combinations, these hybrid architectures, held together mostly by noncovalent interactions, virtually allow the fabrication of any kind of assembly offering a unique opportunity for fine-tuning the properties of materials. However, reproducibility, scale-up, assembly into ordered structures and processability are the challenges yet to be addressed to technologically harness their full potential, and enable their integration into mass produced commercial devices. In this perspective article we analyse the recent developments in the automatised production of hybrid solution processed 2D material heterostructures, especially emphasising on the technologies that are currently closer to achieving low-cost, high-throughput standardised production, namely spray coating, inkjet printing and 3D printing, to sense the direction this research field is taking in pursuit of the development of commercialisable products.
\end{abstract}

\section{Introduction}

Since the discovery of graphene, ${ }^{1}$ the realm of $2 \mathrm{D}$ materials (2DMs) has flourished into a speckled range of chemical compositions including most elements of the periodic table, displaying a rich variety of unique mechanical and electronic properties: $^{2-8}$ graphene, the most renowned, is a zero gap semiconducting, ${ }^{1}$ transparent, extremely strong (i.e., Young's modulus $\sim 1 \mathrm{TPa})^{9}$ yet flexible material; ${ }^{10}$ transition metal dichalcogenides (TMDs) are almost as thin, transparent and flexible as graphene (i.e., elastic moduli $\sim$ a third of graphene), ${ }^{11}$ but as monolayers these are direct bandgap semiconductors (i.e., $1-2 \mathrm{eV}$; in the bulk their band gap is indirect) ${ }^{12,13}$ hexagonal boron nitride (h-BN) is a wide bandgap insulator (5.97 eV, optimal dielectric substrate) $){ }^{14-16}$ with high thermal and chemical stability, and thickness-independent mechanical strength comparable to graphene; ${ }^{17}$ black phosphorous (BP) is characterised by high mobility and thicknessdependent band gap ${ }^{18}(0.3-2 \mathrm{eV}$ from bulk to monolayers $) ;^{19}$ transition metal carbides and nitrides (MXenes) are mostly

Institut für Organische Chemie, Universität Wien, 38 Währinger Strasse, A-1090 Wien, Austria.E-mail: laura.maggini@univie.ac.at metallic (i.e., few systems expected to be semiconductors), ${ }^{20}$ with functionalization-dependent work functions (e.g., from $1.6 \mathrm{eV}$ for $\mathrm{Sc}_{2} \mathrm{C}(\mathrm{OH})_{2}$ to $8.0 \mathrm{eV}$ for $\left.\mathrm{Cr}_{2} \mathrm{CO}_{2}\right)^{21,22}$ and the highest effective Young's modulus upon indentation for solutionprocessed 2DMs (including graphene oxide). ${ }^{23}$ Additionally, the 2DM bidimensional geometry possesses excellent compatibility with currently exploited industrial manufacturing platforms, facilitating direct integration into industrial production processes (e.g., Si production line). ${ }^{24,25}$

2DMs have thus gained great thrust for implementation both in microscopic (e.g., (opto)electronics, photonic devices, sensing, energy storage, etc.) and macroscopic (e.g., composites, catalysis, water remediation, etc.) applications. ${ }^{26-28}$ However, forthcoming technologies based on advanced materials require a stringent yet faceted portfolio of properties (e.g., charge mobility, bandgap, optical transparency, flexibility, etc.), hardly feasible by a single 2DM. The example of graphene is compelling: despite its mechanical strength and flexibility, high charge carrier mobility, and optical transparency, ${ }^{29}$ the lack of a band gap in its electronic structure ${ }^{1}$ clearly prevented its effective implementation, for example, in (opto)electronic applications. To overcome the intrinsic limitations of individual 2DMs, physical and chemical methods to modulate their properties 
(e.g., structural, composition and external field tuning) have been promptly developed. ${ }^{30}$ Nonetheless, despite being effective, these approaches suffer severe reproducibility and scaleup issues, making them incompatible with the requirements for high-throughput product manufacturing.

With the aim of finding accessible, reproducible and nondestructive property-tuning methodologies, the paradigm shifted towards the hybridisation (i.e., vertical or lateral hierarchised stacking) of 2DMs with complementary materials of similar or other dimensionality $(2 \mathrm{D} / 0-1-2-3 \mathrm{D})^{31}$ without chemical bonding, exclusively exploiting van der Waals interactions. This enabled the straightforward prototyping of a kaleidoscopic variety of heterostructures, macro-ordered architectures possessing bespoke composition-dependent physical and mechanical properties (Fig. 1). ${ }^{31,32}$

Specifically, by definition a heterostructure is a hybrid system presenting an interface between two materials. ${ }^{31,32}$ When these heterostructures are composed of two semiconductors with dissimilar band gaps, presenting a coupled interface in which these generate a band alignment or rectify a contact after the equilibration of the Fermi levels (or work functions), they are also defined as "heterojunctions". ${ }^{33}$ These terms have often been used interchangeably and have sometimes generated confusion in the definition of more sophisticated bulk heterostructures (e.g., several heterojunctions, different dimensionality etc.) than the $2 \mathrm{D} /$

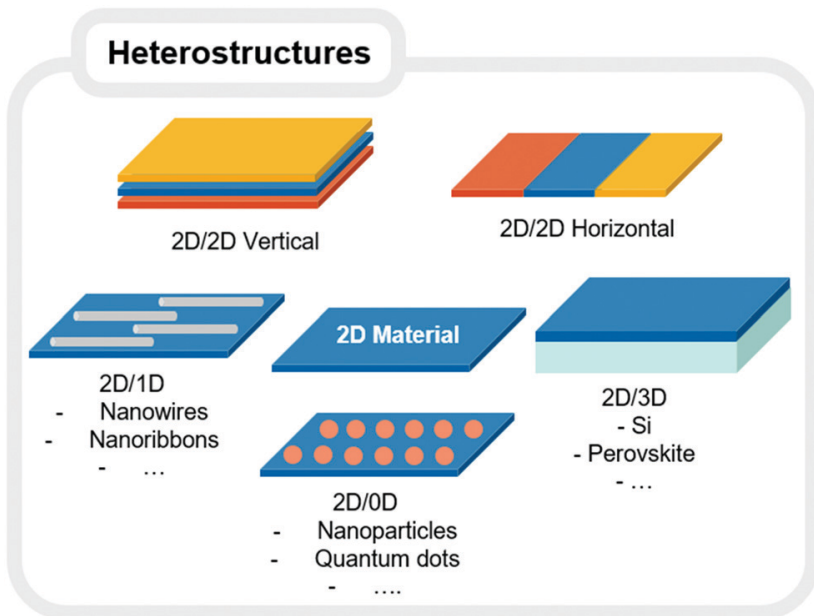

Fig. 1 Schematic representation of the possible 2D material-based van der Waals heterostructures. Reproduced with permission. ${ }^{31}$

2D heterostructures composed of two atomic thin layers for which the terms heterostructure and heterojunction overlap. For the sake of clarity, we will only use the term heterostructure in this manuscript to address the reported hybrid architectures, independently of whether they also comply with the definition of the heterojunction.

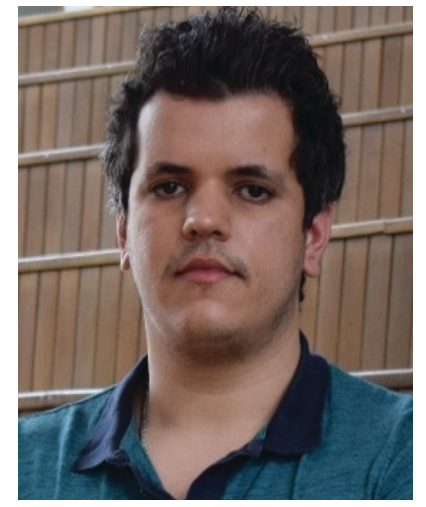

Rúben R. Ferreira
Rúben R. Ferreira obtained his master's degree in Bioorganic Chemistry from the NOVA University of Lisbon in 2016. During his studies, Rúben worked in several projects with the Photochemistry and Supramolecular Chemistry Group. After completing his master's degree, Ruben joined the company Ynvisible - Lisbon, as a Researcher working on the development and characterization of electrochromic displays. During his work, Rúben worked in several European projects and collaborated with many companies, research institutes and universities. Later on, he assumed the position of Project Development Specialist and moved to Freiburg - Germany, where he helped set up the company's new R\&D lab and managed internal and external development projects. In April of 2020, Rúben joined the group of Prof. Bonifazi at the University of Vienna as a PhD candidate, to develop new multi-functional electrochromic materials and devices based on covalent organic frameworks. 
The heterostructure approach, which could virtually lead to any desired combination, thus offers a unique synergistic opportunity to overcome the inherent limitations of each of its components and build tailored architectures at the limit of atomic resolution presenting improved or even unprecedented properties when compared to their constituting elements. The landmark production of graphene field effect transistors (FETs) supported by h-BN by Hone and coworkers in $2010^{14}$ demonstrated that h-BN can serve as a perfect dielectric substrate effectively enabling graphene-based electronics, by reporting charge transport measurements up to $60000 \mathrm{~cm}^{2} \mathrm{~V}^{-1} \mathrm{~s}^{-1}$. Few years later the same group reported the production of FETs with a molybdenum disulfide $\left(\mathrm{MoS}_{2}\right)$ channel, graphene gate and h-BN dielectric on flexible substrates, ${ }^{34}$ presenting mobility $\left(27 \mathrm{~cm}^{2} \mathrm{~V}^{-1} \mathrm{~s}^{-1}\right.$ ) comparable to that attainable on a rigid $\mathrm{SiO}_{2}$ substrate $\left(45 \mathrm{~cm}^{2} \mathrm{~V}^{-1} \mathrm{~s}^{-1}\right)$. Since then a profusion of heterostructures characterised by enhanced synergistic performance were produced, advancing their applicability in fields such as (opto)electronics, sensing, energy storage, catalysis, etc. $^{30-32,35-41}$

The pioneering processes for the prototyping and fundamental investigations of 2DM heterostructures were the topdown mechanical exfoliation and the bottom-up chemical vapour deposition (CVD) techniques. ${ }^{30-32}$ Although producing the highest quality 2DMs, these technologies either lack scalability or remain costly and complex in their execution concerning heterostructure production..$^{30-32}$ For instance, the exfoliation and restacking approach offers great design flexibility, but remains intrinsically not scalable in both size (i.e., $\mu \mathrm{m}$ range) and quantity of $2 \mathrm{DM}$ heterostructures produced. ${ }^{30-32}$ CVD, which requires high temperatures and high vacuum, is hindered by the sensitive growth conditions specific for each 2DM. Such restrictions hamper design flexibility and the growth of quality 2DMs onto prior layers without damage. Moreover, the mandatory requirement of a final transfer step from the growth substrate onto a target substrate might degrade the properties of the heterostructure either by damaging its structure (i.e., introducing defects, wrinkles, etc.), or by contaminating the hybrid's surface (i.e., residuals of polymeric carrier in wet transfer processes). ${ }^{32}$

To nonetheless harness the high quality of CVD produced 2DMs in heterostructures, researchers started to employ this process in combination with other manufacturing technologies (i.e., inkjet printing; vide infra Section 2.2), averting the growth condition incompatibility issue of different 2DMs. Quellmalz, Niklaus and coworkers recently reported a versatile approach for the transfer (i.e., monolayer graphene from copper foils to $10 \mathrm{~cm} \mathrm{Si}$ wafers) and stacking of 2DMs into heterostructures (i.e., graphene/h-BN heterostructures; semiconducting doublelayer graphene) by a resin-mediated "gluing" process (i.e., adhesive bonding), employing solely commercially available wafer bonding equipment. ${ }^{42}$ Specifically, a resin is initially spin coated onto the target substrate $(2.5 \mu \mathrm{m})$, then the CVD grown monolayer of interest (i.e., on the $\mathrm{Cu}$ foil) is placed on top of the target wafer and bonded in a wafer bonder in an inert atmosphere (i.e., for graphene $190{ }^{\circ} \mathrm{C}, 20 \mathrm{~min}, 3 \mathrm{kN}$ ). Follow-up etching (i.e., $\mathrm{FeCl}_{3}$ to remove $\mathrm{Cu}$ foil) and rinsing steps provide the transferred material; to produce heterostructures the process is adapted (i.e., etching step depending on growth substrates) and repeated as many times as the required layers. The proposed methodology definitely represents a substantial advancement in the production of wafer size heterostructures through a process compatible with the high-volume production lines of the semiconductor industry. However, this process remains inherently elaborate, costly in its execution and limited in design flexibility (e.g., expensive masks for design; rigid substrates; etc.).

Accessible solution-processed heterostructures started to be produced via layer-by-layer assembly performed with nonautomated methodologies, such as drop casting, filtration, spin coating, etc. ${ }^{43,44}$ exploiting single or few-layer 2DM suspensions obtained by ultrasonication and intercalation. ${ }^{45}$ Despite the straightforward implementation, the performance of these solution-processed heterostructures was negatively impacted by the lack of exact reproducibility, fine-tuning of their structural parameters (e.g., number of layers, thickness, roughness, etc.) and technical limitations (e.g., vacuum filtration: devicedependent size of the assembly, lengthy process, required transfer procedure, not scalable; spin coating: limited thickness to $50 \mathrm{~nm}$, loss of homogeneity upon scaling up of the coated area, high wastage, not compatible with diluted solutions and flexible substrates) hindering scale up of production. ${ }^{45}$ Satisfactory and reliable performance among large scale productions is an essential requirement for the deployment of 2DMs in commercialisable products. ${ }^{24}$

The formulation of solution processable $2 \mathrm{D}$ materials into inks ${ }^{4,46-49}$ has eventually enabled the controlled (i.e., thickness, dimension, roughness, etc.) "functional" deposition (i.e., structure able to perform a function) of 2DMs, exploiting established printing and coating technologies. This transition towards automatised, low-cost and large-scale manufacturing technologies is foreseen capable of facilitating mass-market adoption of $2 \mathrm{DMs},{ }^{50}$ and is now starting to prove its disruptive advantages for the standardised fabrication of heterostructures, removing the need for developing new manufacturing technologies for these hybrids. Indeed, industrial-scale printing facilities have developed to satisfy the tight cost control of high volume manufacturing industries (e.g., packaging, newspapers, etc.) and are thus optimised for minimal operating costs (e.g., environmental temperature and pressure), swift massproduction (i.e., printing speeds $>100 \mathrm{~m} \mathrm{~min}^{-1}$ ) and compatibility with flexible materials (i.e., paper, plastic, and textiles optimal for flexible and wearable electronics). ${ }^{50}$

Several reviews have been published on $2 \mathrm{D}$ material heterostructures ${ }^{26,30-32,38-41,51-61}$ focusing on material synthesis and characterisation of their chemical, physical, and optoelectronic properties. For state-of-the-art fabrication of $2 \mathrm{D} / 3 \mathrm{D}$ heterostructures, we refer the readers to the critical survey by Kim et al. on the production of $2 \mathrm{D}$ materials onto bulk materials and vice versa. ${ }^{62}$ The scope of this perspective article is instead that of highlighting the recent advances in heterostructure fabrication exploiting industry-friendly 


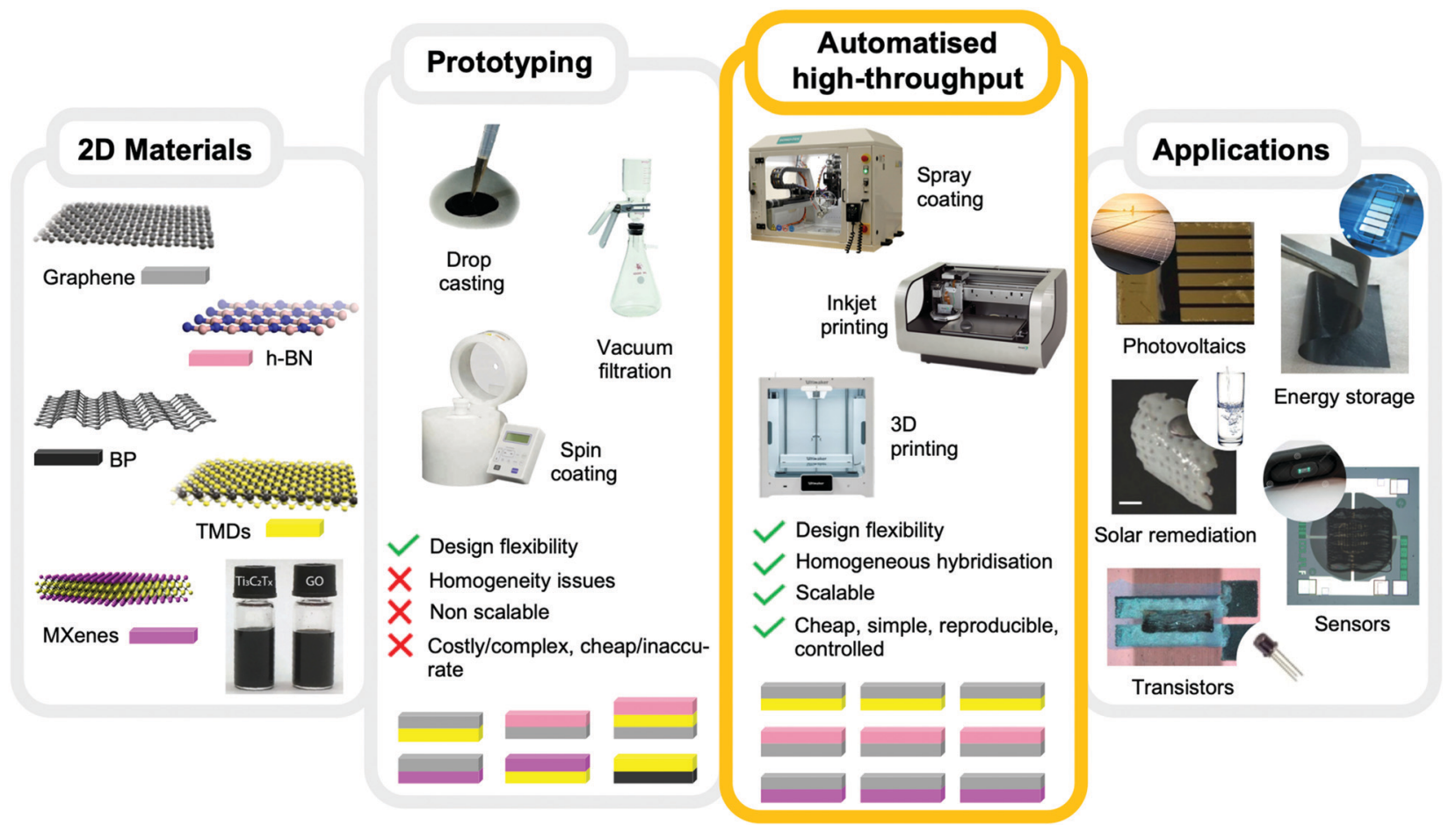

Fig. 2 Schematic representation of the 2D material solution-processed heterostructure roadmap, from left to right: the exfoliation and solution processing of the $2 \mathrm{DMs}$, the pioneering attempts of dry and wet heterostructure assembly which led to the fast prototyping of a kaleidoscopic variety of hybrid hierarchised architectures, the high throughput assembly processes reviewed in this Perspective Article today applied for their large scale standardised manufacturing leading to their integration into mass produced applications. Reproduced with permission. ${ }^{63-69}$

solution-processing technologies (i.e., spray coating, inkjet printing and 3D printing) that are currently being explored for the preparation of heterostructures, paving the way to their automatised, reliable, large-scale production, hinting at their awaited integration in commercial products (Fig. 2).

We herein review the latest scientific breakthroughs reported in the literature concerning the solution processed fabrication of $2 \mathrm{DM}$ heterostructures with coating and printing manufacturing technologies. Upon a thorough bibliographic research we specifically identified mainly three technologies currently thoroughly investigated for the achievement of the ambitious goal of optimising and standardising the manufacturing of $2 \mathrm{D}$ material heterostructure-based products, namely spray casting, inkjet printing and 3D printing. After a brief introduction on the machines, their technological improvements towards the delivery of bespoke heterostructures will be discussed as well as the specifics of the deposition strategy and device design/integration, providing a holistic overview on this research field from a combined engineering/manufacturing, materials science and chemical perspective. The examples reported have been selected not only for the performance improvement detected for the produced heterostructures, but most importantly on the merit of production homogeneity and performance reliability. Where possible, considering the limited amount of currently available literature for this cutting-edge research topic, the focus will be kept on the production of heterostructures tailored for integration into applications within the scope of this journal (e.g., transistors, photovoltaics, sensors, etc.).

\section{Coating and printing technology- based heterostructure fabrication}

The emergence of solution processed 2D materials and ink formulation ${ }^{43,44,46,48,49,70-72}$ has enabled their value-added harnessing into established coating and printing technologies, offering the promise of their automatised (i.e., consistent), scalable manufacturing and device integration (e.g., (opto)electronics, photonics, energy storage, sensing, etc.). ${ }^{4,73}$ Coating and printing technologies, including spray coating ${ }^{74}$ inkjet printing, ${ }^{75}$ and extrusion-based 3D printing, ${ }^{76}$ have evolved to implement $2 \mathrm{DM}$ based device manufacturing with high homogeneity and resolution, by adapting their printing components and processes to 2DM inks. ${ }^{46}$ In parallel, since the first example of inkjet-printed graphene-based flexible electronics reported in $2011,{ }^{77}$ sustainable progress (e.g., shift to non-toxic, volatile solvents, conscious choice, use of additives and binders, etc.) in functional ink formulation enabled optimisation of the 2DM inks' fluidic characteristics to match the printing requirements of the manufacturing platforms (e.g., viscosity, volatility, wettability, viscoelasticity, etc.) facilitating effective deposition, as well as improvement of the drying/adhesion (i.e., resolution) of the printed features. ${ }^{46}$

In this section we will outline the recent achievements in the employment of these technologies for the large-area, highvolume production of functional $2 \mathrm{D}$ material heterostructures (e.g., both layer-by-layer and randomly assembled) and their implementation in the state-of-the-art devices characterised by property consistency advantages. 


\subsection{Spray coating}

Spray coating is a versatile and cost-effective deposition technique suitable for the large-area coating of rigid and flexible substrates. ${ }^{78,79}$ A carrier gas (i.e., $\mathrm{N}_{2}$ ) pushes the liquid ink (viscosity: 1-1000 $\mathrm{mPa} \mathrm{s} ; 2 \mathrm{D}$ material loading: 0.1-10 $\mathrm{w} \%$ ) through a small nozzle towards the substrate. ${ }^{79}$ Once the droplets hit a surface they coalesce into a uniform wet film (resolution: 2-200 $\mu \mathrm{m}$ ), which later solidifies upon evaporation of the solvent. Due to its divergent automatised spraying, a uniform and continuous film can be reproducibly achieved even in substrates with a highly rough surface. Furthermore, this technique allows fine tuning over film thickness and morphology by adjusting carrier gas pressure (size and speed of the droplets), the distance between the nozzle and the substrate, and substrate temperature. ${ }^{79}$

In 2007 Kaner et al. demonstrated the potential of spray casting in the fabrication of highly uniform and semiconducting graphene sheets (i.e., with tailored size, thickness) obtained via the initial deposition of a graphene oxide aqueous dispersion followed by reduction. ${ }^{80}$ The combination of droplet size control and rapid evaporation (i.e., avoidance of the coffee ring effect and evaporation driven concentration), led to homogeneous reproducible films in nearly $100 \%$ yield within hours. This technology has only recently started to be exploited for the controlled production of 2D material heterostructures. In 2019 Gogotsi and coworkers reported the use of an airbrush for the

a)

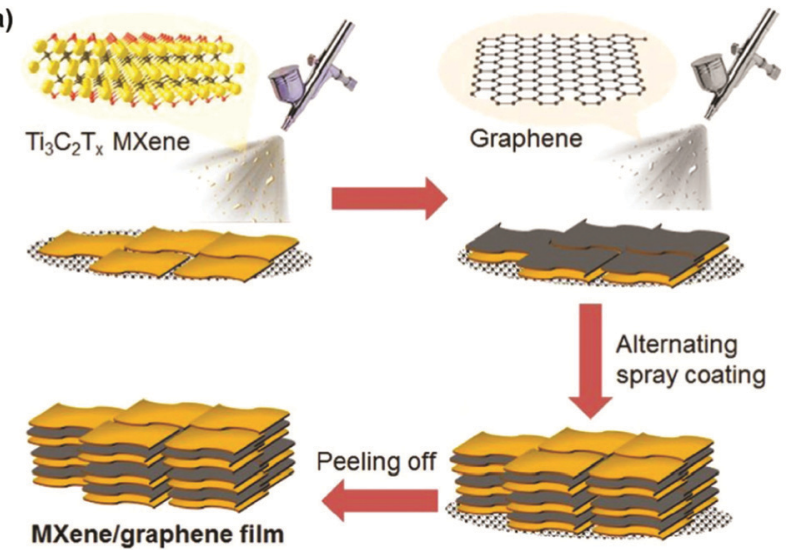

b)

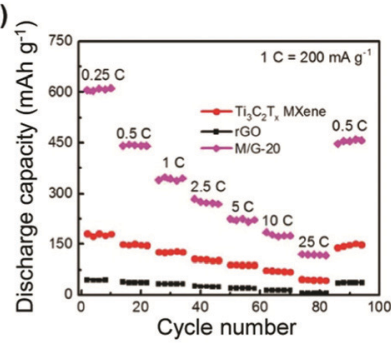

c)

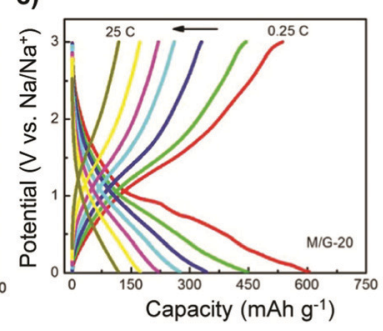

Fig. 3 (a) Schematic representation of the manufacturing of freestanding and flexible 2D MXene/graphene heterostructured films by a spray-assisted LbL process. (b) Rate profiles. (c) Charge-discharge curves of the M/G-20 film at different current rates. Reproduced with permission. ${ }^{63}$ Copyright 2019, John Wiley and Sons. scalable manufacturing of 2D MXene/graphene heterostructured free standing films by spray-assisted layer-by-layer assembly (Fig. 3). ${ }^{63}$ Specifically, by spraying alternating thin layers of $\mathrm{Ti}_{3} \mathrm{C}_{2} \mathrm{~T}_{x}$ MXene and reduced graphene oxide (rGO), the authors obtained a hybrid flexible film with a thickness of $2-3 \mu \mathrm{m}$, combining the energy storage capability of MXenes with the electrical conductivity of graphene. Interestingly, the authors were able to produce $22 \times 28 \mathrm{~cm}$ films within $30 \mathrm{~min}$, indicating the possibility of high throughput fabrication. Moreover, when compared to their individual building blocks, the hybrid structure displayed improved electrochemical performance when used as an anode for Na-ion storage ( $4: 1$ ratio achieved a reversible capacity of $600 \mathrm{~mA} \mathrm{~h} \mathrm{~g}{ }^{-1}$ at $0.25{ }^{\circ} \mathrm{C}>$ pristine $\mathrm{Ti}_{3} \mathrm{C}_{2} \mathrm{~T}_{x}$ MXene $180 \mathrm{~mA} \mathrm{~h} \mathrm{~g}{ }^{-1}$ and rGO films $45 \mathrm{~mA} \mathrm{~h} \mathrm{~g}^{-1}$ ).

In an effort to further improve the homogeneity of spray coated films of 2D materials by facilitating the nebulisation of their inks, ultrasonic spraying systems have been developed. ${ }^{81}$ Unlike conventional pressure nozzles, ultrasonic nozzles utilise piezoelectric transducers to shear the solution and create a fine mist using ultrasonic vibrations. As a result, smaller monodisperse droplets can be achieved $(2-20 \mu \mathrm{m})$, whose size can be modulated by adjusting the vibration frequency, increasing the resolution of the coating process and enhancing the homogeneity of the deposited films. This technology has found ample application in the production of hybrid perovskite solar cells, and specifically for the deposition of the perovskite layer, in combination with spin coating or thermal evaporation for the deposition of the electron and hole transport layers. ${ }^{82,83}$ In 2014 Lidzey et al. reported the fabrication of planar heterostructure $\mathrm{CH}_{3} \mathrm{NH}_{3} \mathrm{PbI}_{3 x} \mathrm{Cl}_{x}$ perovskite solar cells under ambient conditions with a peak power conversion efficiency (PCE) of $11 \%{ }^{84}$ The process involved dissolving perovskite precursors methylammonium iodide (MAI) and $\mathrm{PbCl}_{2}$ powder in DMF (a molar ratio of $3: 1$; total conc. $100 \mathrm{mg} \mathrm{mL}^{-1}$ ), and depositing this precursor ink in a single pass onto a heated pre-patterned glass ITO/PEDOT:PSS (30 nm layer, deposited by spin-coating) affording a dry film, which was later annealed to promote crystallisation of the perovskite.

Choi et al. further enhanced the control and definition of this deposition platform, developing a megasonic spray coating system for the manufacturing of large area hybrid organicinorganic perovskite solar cells (Fig. 4). ${ }^{85}$ The spray system consisted of an ultrasonic nebuliser fitted with a plastic nozzle, attached to a moving arm allowing movement in the $Y$-axis. The substrate was placed on a heated stage capable of moving in the $X$-axis, allowing the continuous deposition of materials over a large area $\left(56.25 \mathrm{~cm}^{2}\right)$. While most ultrasonic spray systems work at frequencies below $1 \mathrm{MHz}$, the system reported by Choi operates at 1.7 MHz, generating homogeneous $2-4 \mu \mathrm{m}$ droplets. A $\mathrm{CH}_{3} \mathrm{NH}_{3} \mathrm{PbI}_{3}$ precursor solution was deposited onto PEDOT:PSS coated glass/ITO using the megasonic spray system, yielding a highly uniform perovskite film after thermal treatment. The perovskite solar cell was fabricated by sequentially depositing via thermal evaporation: $\mathrm{C}_{60}(20 \mathrm{~nm})$, bathocuproine $(10 \mathrm{~nm})$, and $\mathrm{Cu}(50 \mathrm{~nm})$. The produced cell gave a peak PCE of $14.2 \%$ (with an average PCE of $13.7 \%$ ), the highest among 

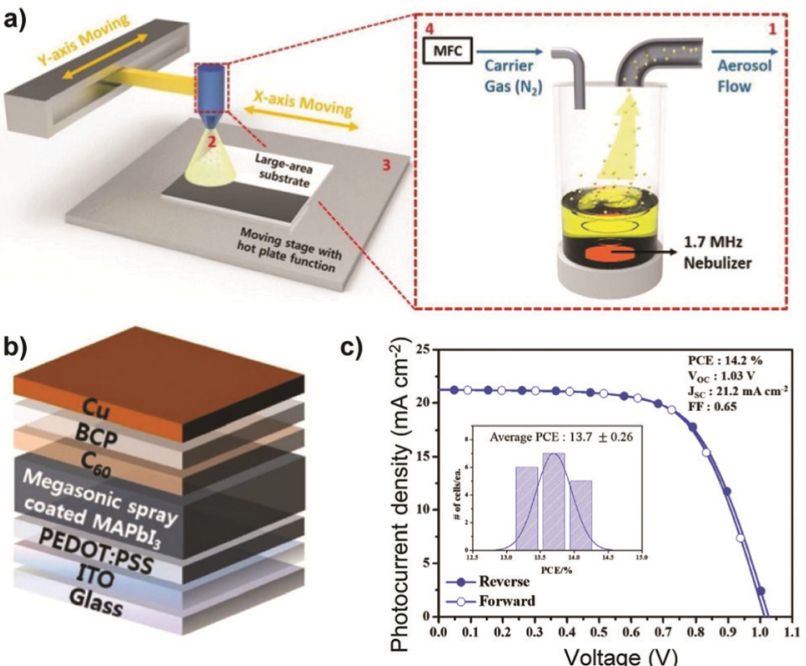

Fig. 4 (a) Schematic representation of the megasonic spray-coating process. (b) Schematic illustration of an inverted planar MAPbI3 perovskite solar cell fabricated via the megasonic spray-coating system. (c) J-V curves and histogram (inset) of 18 megasonic sprayed perovskite solar cells with a $1 \mathrm{~cm}^{2}$ active area under one sun illumination. Reproduced with permission. ${ }^{85}$ Copyright 2018, John Wiley and Sons.

spray-coated large-area (i.e., active area of $10 \mathrm{~mm}^{2}$ ) perovskite solar cells.

Lidzey and coworkers ultimately developed an all spray coating-based process, consisting of the sequential deposition of tin oxide thin films, a triple-cation perovskite and spiroOMeTAD $(\sim 200 \mathrm{~nm}),{ }^{64}$ drastically decreasing the overall production cost without affecting their efficiency (Fig. 5). On small-area substrates $\left(2.5 \mathrm{~mm}^{2}\right)$, the authors achieved a maximum reverse scan PCE of $19.4 \%$, the highest reported to date, with an average PCE of $16.6 \pm 2.4 \%$. On large-area devices $\left(15.4 \mathrm{~mm}^{2}\right), 16.3 \%$ PCE was achieved, with an average of $10.3 \pm$ $4.0 \%$. In this case, the average PCE was hindered by the presence of structural defects in the active area of some devices, indicating that with further optimisation of the coating process higher PCEs could be achieved for this fully spray deposited devices.

\subsection{Inkjet printing}

Inkjet printing is a digital non-contact printing technique vastly used in both research and industry, where ink droplets are jetted and deposited in fast succession onto a heated substrate to produce predesigned patterns without requiring a mask. ${ }^{46,79}$ Characterised by very low ink consumption (pL- $\mu \mathrm{L}$ drops, 1-2 mL; required viscosity: $4-30 \mathrm{mPa}$ s; $2 \mathrm{D}$ material loading: $2-10 \mathrm{w} \%$ ), this technology can provide an attractive route to the fabrication of complex heterostructures with high resolution (i.e., $10-200 \mu \mathrm{m}$ ), low cost, and large scale advantages. ${ }^{47,86,87}$ Based on the droplet generation mechanism, there are two main types of inkjet printing, continuous inkjet printing (C-IJP) and drop-on-demand inkjet printing (DOD-IJP).

In a seminal report, Coleman and coworkers reported a hybrid process involving the inkjet printing of conductive graphene electrodes and spray coating of a dielectric h-BN layer
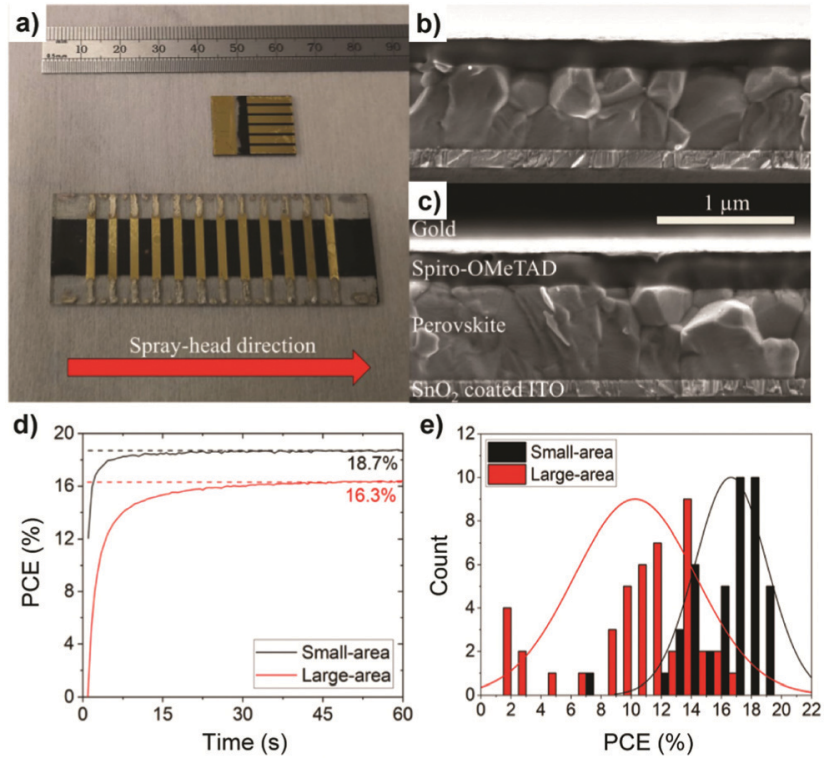

Fig. 5 (a) Small and large-area fully spray-coated perovskite solar cells. (b) Cross-sectional SEM image of complete devices incorporating a spraycast perovskite layer. The device in part (b) utilises spin cast $\mathrm{SnO}_{2}$ and spiro-OMeTAD layers whereas the device in part (c) is fully spray-coated. (d) Output power of the champion devices when held (for $60 \mathrm{~s}$ ) at a fixed voltage close to the maximum power point. (e) Histogram of reverse-scan PCE data from 43 fully spray-cast small-area devices and 45 large-area devices. Reproduced with permission. ${ }^{64}$ Copyright 2020, Springer Nature.

with thicknesses ranging from 1.65 to $5.15 \mu \mathrm{m}$ to produce allprinted capacitors. The devices displayed capacitances ranging from 0.24 to $1.1 \mathrm{nF} \mathrm{cm}{ }^{-2}$, indicating the creation of pinholefree films. ${ }^{88}$ Shortly after, the same group produced all printed transistors composed of vertically stacked nanosheet networks comprising a graphene source, drain, and gate electrodes; a TMD (i.e., $\mathrm{MoS}_{2}, \mathrm{MoSe}_{2}, \mathrm{WS}_{2}$, and $\mathrm{WSe}_{2}$ ) channel; and a h-BN separator. These devices displayed an ON/OFF current ratio of $6 \times 10^{2}$ and a mobility of $0.1 \mathrm{~cm}^{2} \mathrm{~V}^{-1} \mathrm{~s}^{-1}$. Torrisi et al. instead printed graphene h-BN field-effect transistors (FETs) on flexible polyester textiles (Fig. 6). ${ }^{65}$ Specifically, the graphene/h-BN textile FETs $(80 \times 500 \mu \mathrm{m})$ were produced by sequentially printing: a PEDOT:PSS film as the gate electrode $(\sim 6.5 \mu \mathrm{m})$; a h-BN dielectric layer $(\sim 2 \mu \mathrm{m}$, also responsible for smoothing the substrate's residual roughness); a thick graphene channel $(\sim 200 \mathrm{~nm})$; and finally PEDOT:PSS source and drain contacts $(\sim 800 \mathrm{~nm})$. Upon topological investigation, the layers became homogeneous, and did not present any visible pinhole or delaminating area. The average field-effect mobility of these flexible devices resulted in $\mu_{\mathrm{h}} \sim 91 \pm 29 \mathrm{~cm}^{2} \mathrm{~V}^{-1} \mathrm{~s}^{-1}$ and $\mu_{\mathrm{e}} \sim 22 \pm 10 \mathrm{~cm}^{2} \mathrm{~V}^{-1} \mathrm{~s}^{-1}$, respectively; whilst the ON/OFF current ratio was recorded to be $1.23 \pm 0.3$. Finally, thanks to a waterproof polyurethane protective layer on the textiles, these FETs proved resilient to water washings ( $>20$ times, no significant degradation).

Casiraghi and Fiori et al. recently reported another hybrid combined CVD-inkjet printing approach for the production of high performance $\mathrm{MoS}_{2}$-based flexible FETs to exploit the higher control on the properties and purity of CVD grown 2D 


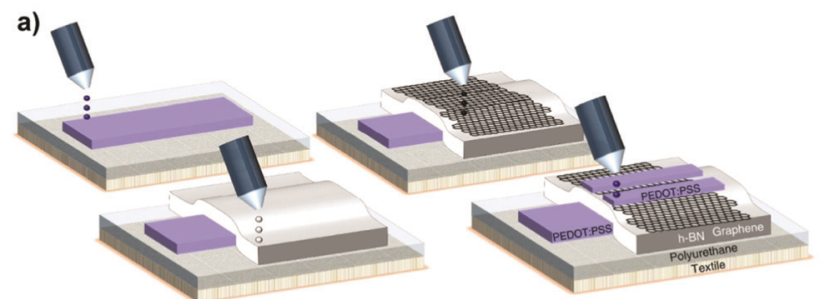

b)


Fig. 6 (a) Fabrication steps of the inkjet-printed inverted-staggered FET heterostructure on textile. (b) Schematic of a printed inverted-staggered FET heterostructure. (c) Inverted-staggered and (d) coplanar FET heterostructures on PET. (e and f) Transfer characteristics of the FETs as a function of $V_{\text {ds. }}$. Reproduced with permission. ${ }^{65}$ Copyright 2017, Springer Nature.

materials. After CVD growth, transfer of $\mathrm{MoS}_{2}$ onto the paper substrate and printing of the h-BN dielectric and silver source/ drain contacts led to devices characterised by an ON/OFF current of $8 \times 10^{3}$ and a mobility of $5.5 \mathrm{~cm}^{2} \mathrm{~V}^{-1} \mathrm{~s}^{-1} .{ }^{89}$ Beyond transistors, Casiraghi et al. formulated functional inkjet-optimised aqueous 2DM-based inks (i.e., graphene, $\mathrm{MoS}_{2}, \mathrm{WS}_{2}$ and h-BN), for the fabrication of fully inkjet-printed heterostructure-based photodetectors on paper showing a responsivity higher than $1 \mathrm{~mA} \mathrm{~W}^{-1}$ and the first ever printed programmable logic device. ${ }^{48}$ In the same year Kaul and coworkers printed graphene/ $\mathrm{MoS}_{2}$ heterostructure-based flexible prosthetic devices comprising a photodetector as a therapeutic remedy for retinal tissue degradation (Fig. 7). ${ }^{90}$ The biocompatible devices (average 98\% cellular viability on the printed substrates) were responsive to incoming broadband visible radiation, and generated a photocurrent that scaled proportionally with the intensity of incident light (photoresponsivity $R \sim 0.30 \mathrm{~A} \mathrm{~W}^{-1}$, and calculated detectivity $D \sim 3.6 \times$ $10^{10}$ Jones).

Fully inkjet-printed graphene/perovskite/graphene heterostructure-based photodetectors have been recently reported by He et al., achieving a photoresponsivity as high as $\sim 0.53 \mathrm{~A} \mathrm{~W}^{-1}$ and a detectivity of $3.4 \times 10^{10}$ Jones in the visible range (400$700 \mathrm{~nm}$ ) at room temperature. ${ }^{91}$ The devices were straightforwardly produced by initial patterned printing of a graphene layer as an interdigitated electrode on glass, followed by printing of a $\mathrm{CH}_{3} \mathrm{NH}_{3} \mathrm{PbI}_{3 x} \mathrm{Cl}_{x}$ perovskite precursor solution and in situ crystallisation (annealing at $90{ }^{\circ} \mathrm{C}$ in a $\mathrm{N}_{2}$ atmosphere).

Hasan and coworkers reported the development of CMOS microplate $(\mu \mathrm{HP})$-integrated graphene/metaloxide breath sensors via inkjet printing, to swiftly (i.e., response and recovery time of
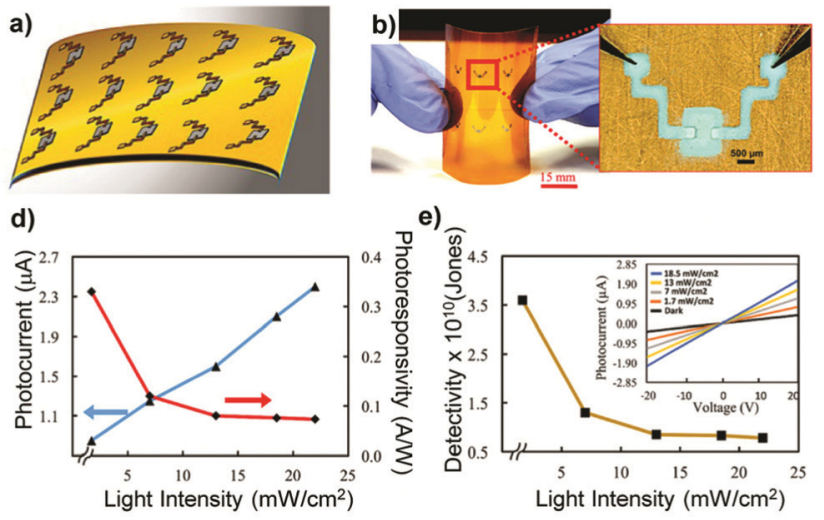

Fig. 7 (a) Representation of the potential for scalability for printing 2D heterostructure devices for AMD over a large format using low-cost inkjet printing on flexible substrates. (b) An actual array of inkjet-printed heterostructure devices on a flexible polyimide film over an area of $60 \times 50 \mathrm{~mm}$. Inset: A single inkjet-printed heterostructure device, where graphene electrodes were printed on top of the $\mathrm{MoS}_{2}$ layer. (c) The dependence of $I_{\mathrm{ph}}$ and $R$ on light intensity at $20 \mathrm{~V}$. (d) $D$ as a function of light intensity at $20 \mathrm{~V}$. Inset: I-V characteristics at various light intensities, where $I_{\mathrm{ph}}$ is seen to increase linearly with light intensity. Reproduced with permission. ${ }^{90}$ Copyright 2017, Springer Nature.

28 and $43 \mathrm{~s}$, respectively) and promptly (i.e., responsivity of $1500 \%$ at $10 \mathrm{ppm}$ pure $\mathrm{NH}_{3}$ ) detect $\mathrm{NH}_{3}$ in exhaled breath, a critical biomarker for a variety of kidney and liver conditions, with excellent cross-device and cross-cycle consistency $(<0.5 \%$ and $<0.41 \%$ variation in responsivity) and long-term stability ( $<1 \%$ variation). ${ }^{66}$ The miniaturised sensing platforms were readily produced by direct printing of a hybrid $\mathrm{ZnO}$-graphene ink onto the electrodes of $\mu \mathrm{HP}(1 \times 1 \mathrm{~mm}$; Fig. 8$)$; 20 passes gave the best condition to optimise trade-off between the uniformity of the printed film, conductivity and reaction/recovery time. This fabrication process enables automated fabrication of multiple devices at once, prompting scalability and device-to-device performance repeatability. Possible interference from acetone was compensated through the parallel deployment of inkjet-printed graphene $/ \mathrm{WO}_{3}$ sensors (superior selectivity towards acetone).

Lin, $\mathrm{Li}$ and coworkers extended the dimensionality of the inkjet printing technology by developing an innovative hybrid 3D freeze-inkjet printing method for the manufacture of a hybrid $\mathrm{MoS}_{2}$ /graphene 3D porous aerogels (Fig. 9). ${ }^{92}$ The procedure described combines inkjet printing with freeze casting, exploiting the formation of ice microcrystals during printing to control both the microstructure and microporosity of the resulting macrostructure. A viscous aqueous ink was prepared by dispersing ammonium thiomolybdate (i.e., $\mathrm{MoS}_{2}$ precursor) and graphene oxide in deionised water. Following a templatedirected approach, this ink was printed onto a Ni foam substrate held at $-30{ }^{\circ} \mathrm{C}$. The ice crystals formed during printing were further grown by placing the substrate in a freezer set at $-70{ }^{\circ} \mathrm{C}$ for $24 \mathrm{~h}$. The aerogel was hence recovered by removal of the ice template through freeze drying, followed by thermal treatment in an inert atmosphere $\left(3 \% \mathrm{H}_{2}, 97 \% \mathrm{Ar}, 2 \mathrm{~h}, 600{ }^{\circ} \mathrm{C}\right)$. Morphological investigations demonstrated that the hybrid aerogel consisted of $\mathrm{MoS}_{2}$ nanoparticles anchored on the 
a)

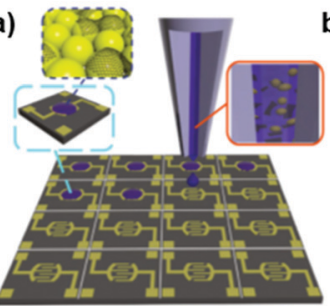

b)
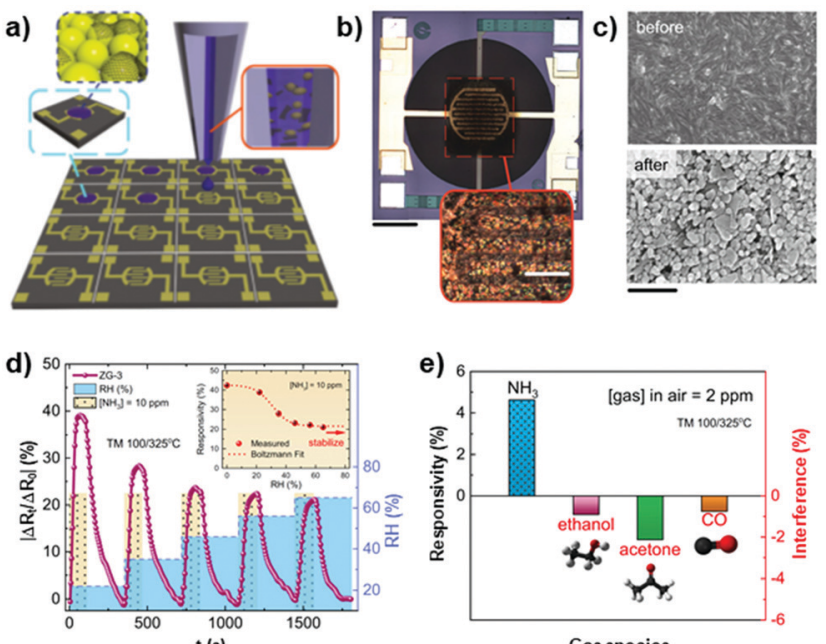

$\mathrm{t}(\mathrm{s})$

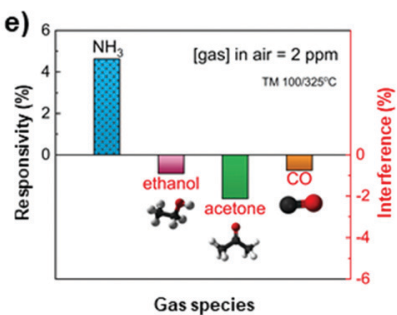

Gas species

Fig. 8 (a) Illustration of the inkjet deposition process. (b) Microscopic image of the inkjet-deposited CMOS sensory device. Scale bar $200 \mu \mathrm{m}$. Inset: Enlarged view: scale bar $20 \mu \mathrm{m}$. (c) SEM images of printed $\mathrm{ZnO}$ graphene composite films: pre-annealing on the top, post-annealing on the bottom. Scale bar $250 \mathrm{~nm}$. (d) Normalized response at $120 / 240 \mathrm{~s} \mathrm{NH}_{3}$ exposure/release cycles varied at various $\mathrm{RH}$ levels; (inset) responsivity as a function of RH levels. (e) Cross-analyte selectivity among common interfering gas species in exhaled breath. Reproduced with permission. ${ }^{66}$ Copyright 2019, Springer Nature.
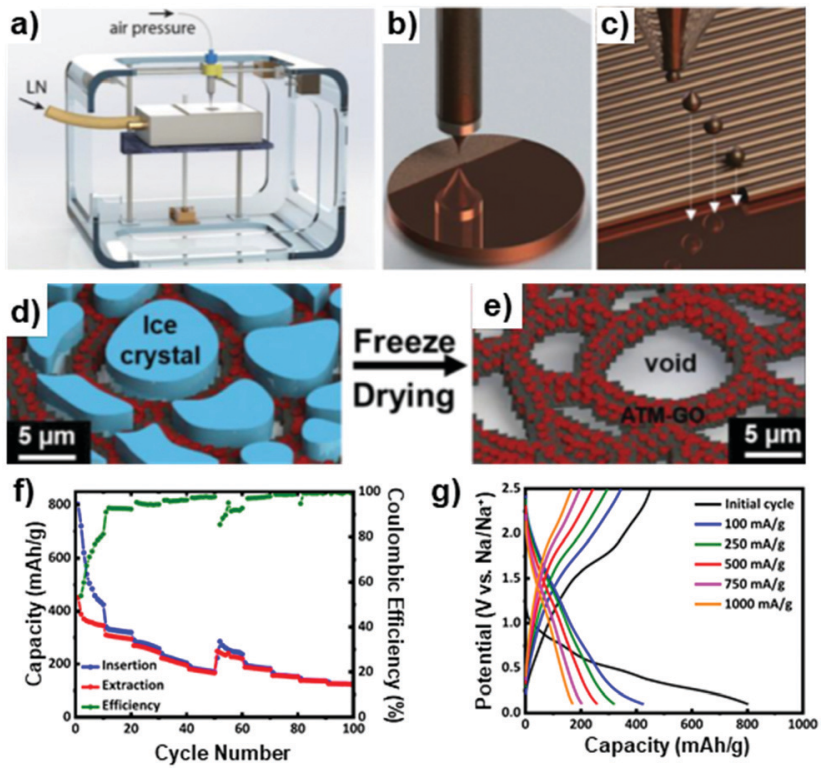

Fig. 9 (a) Schematics of the 3D "drop-on-demand" ink jet printing setup. (b and c) Printing process of the ATM-GO droplets in a raster fashion. (d) Ice template formation during printing. (e) Resulting ATM-GO aerogel after freeze drying. (f) Rate performance at 5 different current density values. (g) Galvanostatic charge-discharge curves of the first cycle in comparison with those in the 10th cycle. Reproduced with permission. ${ }^{92}$ Copyright 2019, Elsevier.

surface of a porous reduced graphene oxide framework. When employed as a porous anode material for $\mathrm{Na}^{+}$ion batteries it reported superior capacitance $\left(\sim 429-800 \mathrm{~mA} \mathrm{~h} \mathrm{~g}^{-1}\right)$ compared

to carbon black/MoS 2 powder electrodes mixed with a polyvinylidene difluoride binder $\left(<200 \mathrm{~mA} \mathrm{~h} \mathrm{~g}{ }^{-1}\right)$ or a $\mathrm{MoS}_{2} /$ reduced graphene oxide aerogel printed on a planar copper foil $\left(<300 \mathrm{~mA} \mathrm{~h} \mathrm{~g}{ }^{-1}\right)$. Lastly, ten Elshof and collaborators reported this year the fabrication of all-inkjet printed solidstate sandwiched supercapacitors composed of $\mathrm{Ti}_{3} \mathrm{C}_{2} \mathrm{~T}_{\mathrm{x}}$ MXene nanosheets as electrodes, and graphene oxide as a solid-state electrolyte (MXene/GO/MXene; device thickness $\sim 4 \mu \mathrm{m}$ ), ${ }^{67}$ in which the proton diffusion is facilitated by the free $\mathrm{H}_{2} \mathrm{O}$ molecules trapped in the graphene oxide layer. The heterostructure-based supercapacitors present high areal capacitance (i.e., $C_{\mathrm{A}}$ of 9.8 and $3 \mathrm{mF} \mathrm{cm} \mathrm{cm}^{-2}$ at a current density of $40 \mu \mathrm{A} \mathrm{cm}^{-2}$, for 30 and 10 MXene layer electrodes respectively), which could be further increased by including liquid electrolytes in the structure, good cycling stability (i.e., $\sim 100 \%$ capacitance retention after 10000 cycles) and high areal energy (i.e., $0.49 \mu \mathrm{W} \mathrm{h} \mathrm{cm}{ }^{-2}$ at a $P_{\mathrm{A}}$ of $12.55 \mu \mathrm{W} \mathrm{cm}{ }^{-2}$ for a 30 MXene layer electrode), comparable with the existing printed supercapacitors.

\subsection{Extrusion-based 3D printing}

Extrusion-based 3D printing is an additive manufacturing technology that offers the possibility of rapidly fabricating (i.e., hours) complex self-supporting three dimensional architectures with tuneable mechanical properties at low cost, with high precision (i.e., resolution: $50-200 \mu \mathrm{m}$ ) and close to zero waste. ${ }^{46,93,94}$ This technique employs a X,Y,Z-motion nozzle or stage to assemble 3D structures by extruding a continuous ink filament at room temperature in a layer-by-layer fashion, upon computerised control. ${ }^{95-97}$ The viscoelastic ink materials (i.e., viscosity: 5-500 $000 \mathrm{mPa}$ s; 2D material loading: 12-40 w\% rapid drying time; self-supporting) must present a shear thinning behaviour to facilitate the extrusion process, and enable shape retention after deposition..$^{98,99}$

By careful design, printed 3D macrostructures of 2DMs can present enhanced mechanical properties, such as high stretchability and negative Poisson's ratio in the case of graphene,${ }^{100,101}$ while retaining the inherent properties of the monolayers (e.g., high electrical/thermal conductivity, surface area, mechanical properties, etc.). Moreover, the applied axial force induced alignment of 2DMs during printing is beneficial for the formation of interconnected 3D networks fostering high thermal/electrical conductivity. ${ }^{95}$ This technology has for example enabled the development of highly stretchable graphenebased electronics, ${ }^{102}$ addressing the issue of conductivity loss of printed planar electronics under large deformations (i.e., low

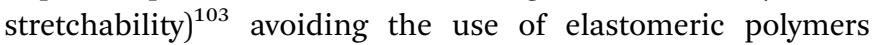
compromising conductivity. ${ }^{104,105}$

Worsley et al. reported in 2015 highly compressible lightweight graphene 3D printed aerogel microlattices, displaying supercompressibility ( $\sim 90 \%$ compressive strain) while maintaining the large surface area of single graphene sheets. ${ }^{106}$ These interconnected microporous architectures proved ideal (e.g., surface area enhancement, porosity, etc.) for integration in energy applications. Indeed, Worsley and $\mathrm{Li}$ et al. reported briefly after the printing of hybrid graphene oxide/graphene 
nanoplatelet/silica nanoparticle composite aerogel electrodes for supercapacitor applications. ${ }^{107}$ These lightweight, conductive porous electrodes exhibited significant electrochemical properties, and the produced supercapacitors (i.e., thickness $\mathrm{mm}$ range) displayed capacitive retentions ( $\sim 90 \%$ from 0.5 to $\left.10 \mathrm{~A} \mathrm{~g}^{-1}\right)$ and power densities $\left(>4 \mathrm{~kW} \mathrm{~kg}^{-1}\right)$ equal or superior to those of 10-100 times thinner devices. These results have recently been improved by the same authors, who reported the $3 \mathrm{D}$ printing of hybrid graphene/ $\mathrm{MnO}_{2}$ porous electrodes favourable for electrolyte and ion diffusion, achieving a record areal capacitance of $44.13 \mathrm{~F} \mathrm{~cm}^{-2}$ and excellent capacitance normalised to area/gravimetry/volume, which is the trade-off for most electrodes (Fig. 10). ${ }^{108}$ These electrodes were produced by homogeneous electrodeposition of $\mathrm{MnO}_{2}$ onto a 3D printed graphene scaffold. The thick (i.e., $4 \mathrm{~mm}$ ) hybrid electrodes reported presented high $\mathrm{MnO}_{2}$ loading (i.e., $182.2 \mathrm{mg} \mathrm{cm}{ }^{-2}$ ), an increase in surface area upon electrodeposition and an almost 4-fold increase in areal capacitance compared to thinner devices (i.e., $44.13 \mathrm{~F} \mathrm{~cm}^{-2}$ and $11.55 \mathrm{~F} \mathrm{~cm}^{-2}$ at $0.5 \mathrm{~mA} \mathrm{~cm}^{-2}$ for 4 and $1 \mathrm{~mm}$ thick electrodes, respectively).

Still in the field of energy related materials, Rocha, GarcíaTunón and collaborators reported the multi-material 3D printing of hybrid graphene/Cu electrodes for energy storage. ${ }^{109}$ In this case both the active material (i.e., graphene oxide) and the current collector (i.e., $\mathrm{Cu}$ ) precursors are formulated into inks and printed into the multicomponent electrode using two

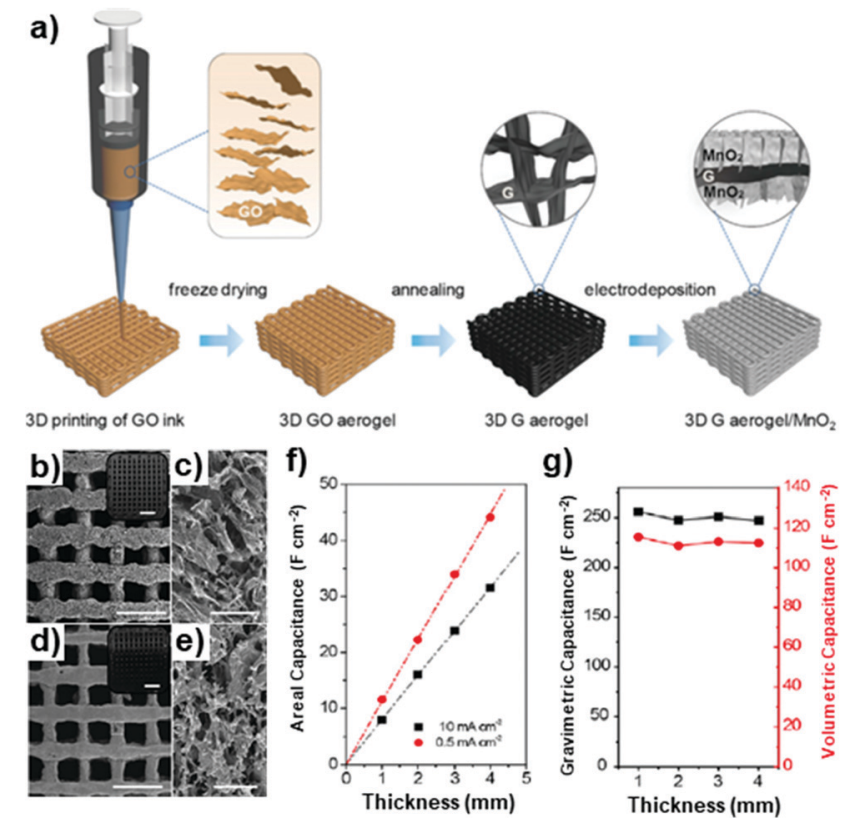

Fig. 10 (a) Schematic illustration of fabrication of a 3D printed graphene aerogel $/ \mathrm{MnO}_{2}$ electrode. (b) Top-view SEM image of a 3D printed graphene aerogel lattice. (c) Magnification of a cylindrical rod highlighted in (b). (d) Top-view SEM image of a 3D printed graphene aerogel lattice electrodeposited with $\mathrm{MnO}_{2}$ for 600 s. (e) Magnification of a cylindrical rod highlighted in (d). (f) Areal capacitance of the electrodes measured at 0.5 and $10 \mathrm{~mA} \mathrm{~cm}^{-2}$. (g) Gravimetric capacitance and volumetric capacitances as a function of electrode thickness. Reproduced with permission. ${ }^{108}$ Copyright 2019, Elsevier. printing nozzles per layer. After printing, the electrodes were sequentially frozen (liquid $\mathrm{N}_{2}$ ), freeze-dried and thermally reduced, providing a self-standing binder-free interdigitated porous electrode $(\sim 5 \mathrm{~mm}$ thick), characterised by specific energy and power density values of $26 \mathrm{~W} \mathrm{~h} \mathrm{~kg} \mathrm{~W}^{-1}$ (at a power density of $1.7 \mathrm{~kW} \mathrm{~kg}^{-1}$ ) and $13 \mathrm{~kW} \mathrm{~kg}^{-1}$ (at an energy density of $1.2 \mathrm{~W} \mathrm{~h} \mathrm{~kg} \mathrm{~W}^{-1}$ ), respectively. The capacitance retention at $15 \mathrm{~A} \mathrm{~g}^{-1}$ resulted in $\sim 80 \%$ after 10000 cycles, indicating promising viability and long-term stability.

For a different application, Fan et al. reported the printing of graphitic carbon nitride $\left(\mathrm{g}-\mathrm{C}_{3} \mathrm{~N}_{4}\right)$ hybrid aerogel membranes with patterned macroscopic architectures to be employed in solar activated wastewater remediation processes (Fig. 11). ${ }^{68}$ The functional ink was formulated by mixing $g-\mathrm{C}_{3} \mathrm{~N}_{4}$ nanosheets (CNNS) with sodium alginate (SA), to achieve correct viscosity and shear thinning behaviour. In the presence of multivalent cations (e.g., $\left.\mathrm{Ca}^{2+}\right)$, SA undergoes a fast gelation reaction by ionically cross-linking the linear chains, resulting in a hydrogel. The authors exploited this reaction to fully cross-link $\mathrm{SA}$ in the $3 \mathrm{D}$ printed samples by submerging the structures in a $\mathrm{CaCl}_{2}$ solution, followed by supercritical $\mathrm{CO}_{2}$ drying to afford the carbon nitride-based hybrid aerogel membranes, characterised by a surface area of $74.2 \mathrm{~m}^{2} \mathrm{~g}^{-1}$ (mean pore diameter $17.81 \mathrm{~nm}$ and volume $0.3303 \mathrm{~cm}^{3} \mathrm{~g}^{-1}$ ). These 3D printed aerogels were then explored for solar wastewater remediation by preparing a CNNS/SA/Au hybrid, in which cetyltrimethylammonium bromide (CTAB)-capped Au nanobipyramids (i.e., $1 \mathrm{wt} \%$ ) were introduced in the aerogel to act as visible light sensitisers and cocatalysts. The ink was prepared in

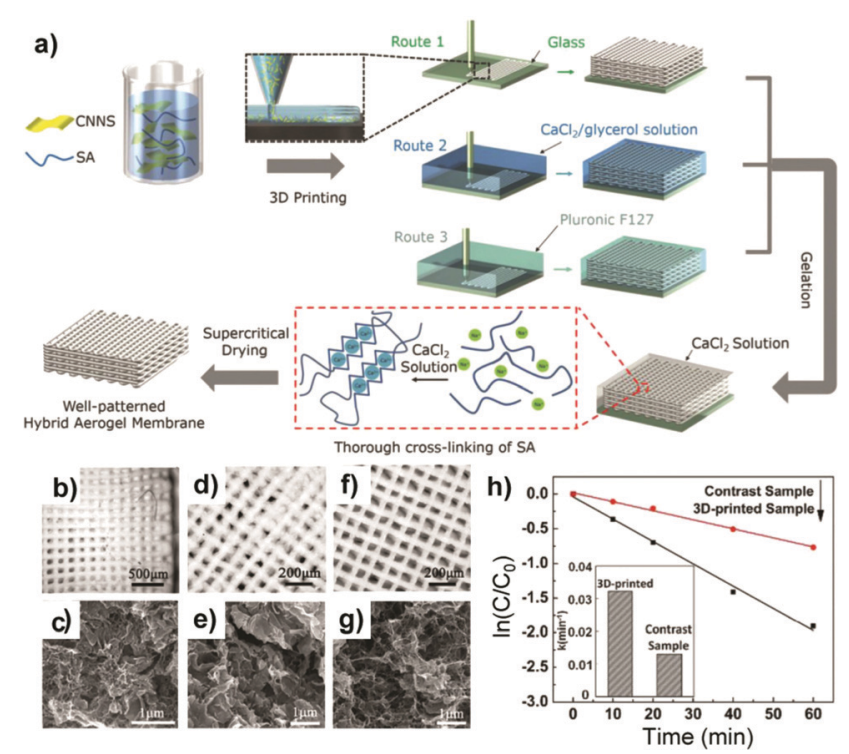

Fig. 11 (a) Schematic illustration of the fabrication process. (b) Optical image of the CN-SA hybrid aerogel membrane printed directly in air and the cross-sectional SEM image (c). (d) Optical image showing the top view of the woodpile structure printed in a $\mathrm{CaCl}_{2} /$ glycerol solution and the cross-sectional SEM image (e). (f) Optical image of a woodpile structure printed in Pluronic F127 and the cross-sectional SEM image (g). (h) Comparison of the photocatalytic MB degradation rates $(\lambda>420 \mathrm{~nm})$. Reproduced with permission. ${ }^{68}$ Copyright 2018, John Wiley and Sons. 
the same manner, by mixing CNNS/Au with SA. The solar wastewater remediation activity was evaluated by testing the methylene blue photodegradation under visible light irradiation $(\lambda>420 \mathrm{~nm})$. This activity was compared to a contrast sample, prepared with the same ink and having equal surface area and mesoporosity, but without the 3D features. The 3D printed sample displayed a superior solar wastewater remediation activity $\left(0.0322 \mathrm{~min}^{-1}\right)$, about 2.5 times higher than the contrast sample, which can be explained by an increase in liquid velocity and dye diffusion. Furthermore, no noticeable decrease in activity was observed after three cycles.

\section{Summary and prospects}

The last decade has witnessed the conceptualisation and thriving of 2DM heterostructures, an exciting playground that stimulated the fantasy of researchers in creating exotic combinations of 2D materials in pursuit of high-performance synergistic outcomes. In this short perspective paper, we have introduced the readers to 2DM heterostructures emphasising their technological potential and the challenges they face with respect to their exploitation in end-user products. After briefly summarising the pioneering heterostructure fabrication processes, with their achievements and limitations, we then focused on discussing the recent progress in the automatised and reliable production of bespoke heterostructure-based devices exploiting solution processed 2DMs and industrially relevant manufacturing platforms, namely: spray coating, inkjet printing and extrusion-based 3D printing. As discussed in the previous section, these three technologies are the ones currently most investigated and promising for the production of 2DM heterostructures. All of these technologies allow high precision deposition of $2 \mathrm{DM}$ inks, when formulated in the appropriate $2 \mathrm{D}$ material loading/viscosity range (see Table 1, no 2DM related limitations). Moreover, these technologies complement each other: spray casting is better at delivering large area films of controlled thickness and roughness; inkjet, on the other hand, is better suited at tackling smaller yet sophisticated patterned architectures; and extrusion-based 3D printing can instead deliver shape-retaining self-supporting 3D architectures, eventually presenting enhanced mechanical properties, depending on the adopted design.

These established technologies, characterised by accessibility, low costs and low consumption merits, have developed adopting to the requirements of 2DM-based device production both in the hardware (e.g., ultrasonic nozzle for spray coaters, size of nozzles) and ink processing (e.g., passes, operative distances, roughness control, etc.), tackling critical controversies concerning the standardisation and prospective mass production of 2DM heterostructure products, achieving:

(I) Strict performance tailoring and control through automation: the development of fully automated (i.e., all sprayed/ printed) production processes not only reduce manipulation steps and production time, but facilitate the controlled (e.g., thickness, roughness, pattern, etc.) and reproducible ordered deposition of 2DMs with high-resolution merits. This control results in the standardisation of the products and of their performances, enabling the definition of defined structureproperty correlations underpinning their performance, to be later employed for the design of application-tailored highly efficient 2DM heterostructures.

(II) Enhanced production sustainability: compared to conventional energy intensive and waste producing manufacturing processes (e.g., CVD requiring high temperature and vacuum, several etching steps, etc.), the manufacturing technologies discussed in this perspective do not operate under fringe conditions, and most importantly do not produce waste. The material needed for the production of the heterostructure is sprayed/printed on demand where needed, without misuse. These inherent technological advantages, combined with novel formulation (e.g., volatile solvents, conscious fillers, additives, etc.) and synthetic paradigms (i.e., synthesis of 2DMs from industrial waste, as bio-based graphene synthesised from lignin recovered from the paper manufacturing industry) will play an important role in further reducing the ecological footprint of this technology.

(III) Scale-up at reduced costs: as mentioned previously, these technologies have already been optimised to satisfy the stringent cost control required in high-volume manufacturing landscapes.

The exciting results achieved hint at the disruptive potential this approach entails, yet further improvements in controlling the manufacturing processes are still needed, and commercialisation will benefit from the standardisation of quality and performance facilitated by these technologies. However, in combination with the improvement of the manufacturing platforms and processes, researchers are also still tackling the absence of standardised, scalable production processes for monolayered materials, which also play a crucial role in the creation of precisely controlled hybrid architectures, and tailoring of their structure/interface-dependent unique properties.

Table 1 Comparison among the coating and printing technologies reviewed in this manuscript

\begin{tabular}{|c|c|c|c|}
\hline & Spray coating & Inkjet printing & 3D printing \\
\hline 2DMs loading & $0.1-10 w t \%$ & $2-10 \mathrm{wt} \%$ & $12-40 \mathrm{wt} \%$ \\
\hline Viscosity & $1-1000 \mathrm{mPa} \mathrm{s}$ & $4-30 \mathrm{mPa} \mathrm{s}$ & $5-500000 \mathrm{mPa} \mathrm{s}$ \\
\hline Resolution & $2-200 \mu \mathrm{m}$ & $10-200 \mu \mathrm{m}$ & $50-200 \mu \mathrm{m}$ \\
\hline Advantages & $\begin{array}{l}\text { Fine-tuning over film thickness } \\
\text { and roughness over large areas, speed }\end{array}$ & Advanced patterning capability & $\begin{array}{l}\text { Tuning of the mechanical } \\
\text { properties of the 3D structures, speed }\end{array}$ \\
\hline Disadvantages & Low patterning resolution & Low speed & Low patterning resolution \\
\hline
\end{tabular}


Indeed, a consistent and high-yield production methodology for individual, large-area, atomically defined 2DM building blocks is currently still unavailable. Further research on the optimisation of the current top-down solution processing methodologies (e.g., homogenisation), the adaptation of established bottom-up synthetic methodologies (i.e., CVD) or the development of novel inexpensive bottom-up synthetic methodologies for 2D monolayered materials (e.g., a chemical approach for $2 \mathrm{D}$ polymers, COFs, etc.) are required in order to achieve highly performant functional inks.

With the acceleration and establishment of reliable, costeffective, large-volume fabrication methods for 2DM heterostructures and the expected quality enhancement of the exfoliated 2DMs, the most conceivable roadmap for the technological exploitation of 2DM heterostructures is inevitably intertwining and accelerated by that of single 2D materials. As forecast for graphene, ${ }^{25}$ it is highly probable that 2DM heterostructure-based technologies will not abruptly disrupt the market, but rather mature within niche applications for which these hybrids will offer unique advantages. The automatised high-throughput production technologies involved will most probably jump-start 2DM heterostructure market integration (i.e., current decade), targeting directly mediumsized markets (e.g., automatised and standardised production) bringing upon radical innovations in the development of marketable 2D material-based devices (e.g., printable electronics, photovoltaics, energy storage, sensing, etc.).

\section{Author contributions}

LM contributed to the conceptualisation of the message brought forward by this perspective article, the literature search supporting it and its writing. RF contributed to the literature search reviewed in this perspective, writing and editing of the images present in this manuscript.

\section{Conflicts of interest}

There are no conflicts to declare.

\section{Acknowledgements}

LM and RF gratefully acknowledge the University of Vienna for financial support, and the European Union's Horizon 2020 research and innovation programme for funding under the Marie Skłodowska-Curie grant agreement no. 101008237 - VIT (polymer engineering via molecular design: embedding electrical and optical properties into VITrimers). The authors thank Dr Vivek Wakchaure for discussion.

\section{Notes and references}

1 K. S. Novoselov, A. K. Geim, S. V. Morozov, D. Jiang, Y. Zhang, S. V. Dubonos, I. V. Grigorieva and A. A. Firsov, Science, 2004, 306, 666-669.
2 S. Z. Butler, S. M. Hollen, L. Cao, Y. Cui, J. A. Gupta, H. R. Gutiérrez, T. F. Heinz, S. S. Hong, J. Huang, A. F. Ismach, E. Johnston-Halperin, M. Kuno, V. V. Plashnitsa, R. D. Robinson, R. S. Ruoff, S. Salahuddin, J. Shan, L. Shi, M. G. Spencer, M. Terrones, W. Windl and J. E. Goldberger, ACS Nano, 2013, 7, 2898-2926.

3 A. G. Kelly, T. Hallam, C. Backes, A. Harvey, A. S. Esmaeily, I. Godwin, J. Coelho, V. Nicolosi, J. Lauth, A. Kulkarni, S. Kinge, L. D. A. Siebbeles, G. S. Duesberg and J. N. Coleman, Science, 2017, 356, 69-73.

4 Z. Zhang, X. Liu, J. Yu, Y. Hang, Y. Li, Y. Guo, Y. Xu, X. Sun, J. Zhou and W. Guo, Wiley Interdiscip. Rev.: Comput. Mol. Sci., 2016, 6, 324-350.

5 M. Naguib and Y. Gogotsi, Acc. Chem. Res., 2015, 48, 128-135.

6 H. Zhang, ACS Nano, 2015, 9, 9451-9469.

7 M. Xu, T. Liang, M. Shi and H. Chen, Chem. Rev., 2013, 113, 3766-3798.

8 A. K. Geim and K. S. Novoselov, Nat. Mater., 2007, 6, 183-191.

9 C. Lee, X. Wei, J. W. Kysar and J. Hone, Science, 2008, 321, 385-388.

10 F. Bonaccorso, Z. Sun, T. Hasan and A. C. Ferrari, Nat. Photonics, 2010, 4, 611-622.

11 S. Bertolazzi, J. Brivio and A. Kis, ACS Nano, 2011, 5, 9703-9709.

12 W. Choi, N. Choudhary, G. H. Han, J. Park, D. Akinwande and Y. H. Lee, Mater. Today, 2017, 20, 116-130.

13 K. F. Mak, C. Lee, J. Hone, J. Shan and T. F. Heinz, Phys. Rev. Lett., 2010, 105, 136805.

14 C. R. Dean, A. F. Young, I. Meric, C. Lee, L. Wang, S. Sorgenfrei, K. Watanabe, T. Taniguchi, P. Kim, K. L. Shepard and J. Hone, Nat. Nanotechnol., 2010, 5, 722-726.

15 J. Xue, J. Sanchez-Yamagishi, D. Bulmash, P. Jacquod, A. Deshpande, K. Watanabe, T. Taniguchi, P. JarilloHerrero and B. J. Leroy, Nat. Mater., 2011, 10, 282-285.

16 K. Watanabe, T. Taniguchi and H. Kanda, Nat. Mater., 2004, 3, 404-409.

17 A. Falin, Q. Cai, E. J. G. Santos, D. Scullion, D. Qian, R. Zhang, Z. Yang, S. Huang, K. Watanabe, T. Taniguchi, M. R. Barnett, Y. Chen, R. S. Ruoff and L. H. Li, Nat. Commun., 2017, 8, 15815.

18 B. Deng, V. Tran, Y. Xie, H. Jiang, C. Li, Q. Guo, X. Wang, H. Tian, S. J. Koester, H. Wang, J. J. Cha, Q. Xia, L. Yang and F. Xia, Nat. Commun., 2017, 8, 14474.

19 V. Tran, R. Soklaski, Y. Liang and L. Yang, Phys. Rev. B: Condens. Matter Mater. Phys., 2014, 89, 235319.

20 H. Kim and H. N. Alshareef, ACS Mater. Lett., 2020, 2, 55-70.

21 Y. Liu, H. Xiao and W. A. Goddard, J. Am. Chem. Soc., 2016, 138, 15853-15856.

22 M. Khazaei, M. Arai, T. Sasaki, A. Ranjbar, Y. Liang and S. Yunoki, Phys. Rev. B: Condens. Matter Mater. Phys., 2015, 92, 75411.

23 A. Lipatov, H. Lu, M. Alhabeb, B. Anasori, A. Gruverman, Y. Gogotsi and A. Sinitskii, Sci. Adv., 2018, 4, eaat0491. 
24 Moving towards the market, Nat. Mater., 2019, 18(6), 519, DOI: 10.1038/s41563-019-0394-4.

25 D. Neumaier, S. Pindl and M. C. Lemme, Nat. Mater., 2019, 18, 525-529.

26 N. R. Glavin, R. Rao, V. Varshney, E. Bianco, A. Apte, A. Roy, E. Ringe and P. M. Ajayan, Adv. Mater., 2020, 32, 1904302.

27 K. Khan, A. K. Tareen, M. Aslam, R. Wang, Y. Zhang, A. Mahmood, Z. Ouyang, H. Zhang and Z. Guo, J. Mater. Chem. C, 2020, 8, 387-440.

28 C. Tan, X. Cao, X. J. Wu, Q. He, J. Yang, X. Zhang, J. Chen, W. Zhao, S. Han, G. H. Nam, M. Sindoro and H. Zhang, Chem. Rev., 2017, 117, 6225-6331.

29 A. C. Ferrari, F. Bonaccorso, V. Fal'ko, K. S. Novoselov, S. Roche, P. Bøggild, S. Borini, F. H. L. Koppens, V. Palermo, N. Pugno, J. A. Garrido, R. Sordan, A. Bianco, L. Ballerini, M. Prato, E. Lidorikis, J. Kivioja, C. Marinelli, T. Ryhänen, A. Morpurgo, J. N. Coleman, V. Nicolosi, L. Colombo, A. Fert, M. Garcia-Hernandez, A. Bachtold, G. F. Schneider, F. Guinea, C. Dekker, M. Barbone, Z. Sun, C. Galiotis, A. N. Grigorenko, G. Konstantatos, A. Kis, M. Katsnelson, L. Vandersypen, A. Loiseau, V. Morandi, D. Neumaier, E. Treossi, V. Pellegrini, M. Polini, A. Tredicucci, G. M. Williams, B. Hee Hong, J. H. Ahn, J. Min Kim, H. Zirath, B. J. Van Wees, H. Van Der Zant, L. Occhipinti, A. Di Matteo, I. A. Kinloch, T. Seyller, E. Quesnel, X. Feng, K. Teo, N. Rupesinghe, P. Hakonen, S. R. T. Neil, Q. Tannock, T. Löfwander and J. Kinaret, Nanoscale, 2015, 7, 4598-4810.

30 M. Zeng, Y. Xiao, J. Liu, K. Yang and L. Fu, Chem. Rev., 2018, 118, 6236-6296.

31 P. Solís-Fernández, M. Bissett and H. Ago, Chem. Soc. Rev., 2017, 46, 4572-4613.

32 Y. Liu, N. O. Weiss, X. X. Duan, H. C. Cheng, Y. Huang and X. X. Duan, Nat. Rev. Mater., 2016, 1, 16042.

33 J. Su, G. Li, X.-H. Li and J.-S. Chen, Adv. Sci., 2019, 6, 1801702.

34 G. H. Lee, Y. J. Yu, X. Cui, N. Petrone, C. C. H. Lee, M. S. Choi, D. Y. Lee, C. C. H. Lee, W. J. Yoo, K. Watanabe, T. Taniguchi, C. Nuckolls, P. Kim and J. Hone, ACS Nano, 2013, 7, 7931-7936.

35 E. Pomerantseva and Y. Gogotsi, Nat. Energy, 2017, 2, 17089.

36 F. Shahzad, M. Alhabeb, C. B. Hatter, B. Anasori, S. Man Hong, C. M. Koo and Y. Gogotsi, Science, 2016, 353, 1137-1140.

37 K. S. Novoselov, A. Mishchenko, A. Carvalho and A. H. Castro Neto, Science, 2016, 353, aac9439.

38 S. Das, J. A. Robinson, M. Dubey, H. Terrones and M. Terrones, Annu. Rev. Mater. Res., 2015, 45, 1-27.

39 X. Huang, C. Tan, Z. Yin and H. Zhang, Adv. Mater., 2014, 26, 2185-2204.

40 H. Wang, F. Liu, W. Fu, Z. Fang, W. Zhou and Z. Liu, Nanoscale, 2014, 6, 12250-12272.

41 A. K. Geim and I. V. Grigorieva, Nature, 2013, 499, 419-425. 42 A. Quellmalz, X. Wang, S. Sawallich, B. Uzlu, M. Otto, S. Wagner, Z. Wang, M. Prechtl, O. Hartwig, S. Luo,
G. S. Duesberg, M. C. Lemme, K. B. Gylfason, N. Roxhed, G. Stemme and F. Niklaus, Nat. Commun., 2021, 12, 917.

43 F. Withers, H. Yang, L. Britnell, A. P. Rooney, E. Lewis, A. Felten, C. R. Woods, V. Sanchez Romaguera, T. Georgiou, A. Eckmann, Y. J. Kim, S. G. Yeates, S. J. Haigh, A. K. Geim, K. S. Novoselov and C. Casiraghi, Nano Lett., 2014, 14, 3987-3992.

44 S. Witomska, T. Leydecker, A. Ciesielski and P. Samorì, Adv. Funct. Mater., 2019, 29, 1901126.

45 X. Cai, Y. Luo, B. Liu and H.-M. Cheng, Chem. Soc. Rev., 2018, 47, 6224-6266.

46 K. Hassan, M. J. Nine, T. T. Tung, N. Stanley, P. L. Yap, H. Rastin, L. Yu and D. Losic, Nanoscale, 2020, 12, 19007-19042.

47 G. Hu, J. Kang, L. W. T. Ng, X. Zhu, R. C. T. Howe, C. G. Jones, M. C. Hersam and T. Hasan, Chem. Soc. Rev., 2018, 47, 3265-3300.

48 D. McManus, S. Vranic, F. Withers, V. Sanchez-Romaguera, M. Macucci, H. Yang, R. Sorrentino, K. Parvez, S. K. Son, G. Iannaccone, K. Kostarelos, G. Fiori and C. Casiraghi, Nat. Nanotechnol., 2017, 12, 343-350.

49 J. N. Coleman, M. Lotya, A. O'Neill, S. D. Bergin, P. J. King, U. Khan, K. Young, A. Gaucher, S. De, R. J. Smith, I. V. Shvets, S. K. Arora, G. Stanton, H. Y. Kim, K. Lee, G. T. Kim, G. S. Duesberg, T. Hallam, J. J. Boland, J. J. Wang, J. F. Donegan, J. C. Grunlan, G. Moriarty, A. Shmeliov, R. J. Nicholls, J. M. Perkins, E. M. Grieveson, K. Theuwissen, D. W. McComb, P. D. Nellist and V. Nicolosi, Science, 2011, 331, 568-571.

50 R. Howe, Printing with graphene: exploring the inks set to unleash graphene's potential, https:/www.mewburn.com/ news-insights/printing-with-graphene-unleashing-graphenespotential-through-mass-production.

51 E. Pomerantseva and Y. Gogotsi, Nat. Energy, 2017, 2, 17089.

52 F. H. L. Koppens, T. Mueller, P. Avouris, A. C. Ferrari, M. S. Vitiello and M. Polini, Nat. Nanotechnol., 2014, 9, 780-793.

53 B. V. Lotsch, Annu. Rev. Mater. Res., 2015, 45, 85-109.

54 M. Y. Li, C. H. Chen, Y. Shi and L. J. Li, Mater. Today, 2016, 19, 322-335.

55 J. Lee, J.-H. Shin, G.-H. Lee and C.-H. Lee, Nanomaterials, 2016, 6, 193.

56 F. Di Maria, M. Zangoli, I. E. Palam, E. Fabiano, A. Zanelli, M. Monari, A. Perinot, M. Caironi, V. Maiorano, A. Maggiore, M. Pugliese, E. Salatelli, G. Gigli, I. Viola and G. Barbarella, Adv. Funct. Mater., 2016, 26, 6970-6984.

57 D. L. Duong, S. J. Yun and Y. H. Lee, ACS Nano, 2017, 11, 11803-11830.

58 I. V. Sankar, J. Jeon, S. K. Jang, J. H. Cho, E. Hwang and S. Lee, NANO, 2019, 14, 1930009.

59 H. Liu, L. Li, M. E. Scofield and S. S. Wong, APL Mater., 2015, 3, 080701.

60 F. Shahzad, M. Alhabeb, C. B. Hatter, B. Anasori, S. Man Hong, C. M. Koo and Y. Gogotsi, Science, 2016, 353, 1137-1140. 
61 K. S. Novoselov, A. Mishchenko, A. Carvalho and A. H. Castro Neto, Science, 2016, 353, 461.

62 S. H. Bae, H. Kum, W. Kong, Y. Kim, C. Choi, B. Lee, P. Lin, Y. Park and J. Kim, Nat. Mater., 2019, 18, 550-560.

63 M. Zhao, N. Trainor, C. E. Ren, M. Torelli, B. Anasori and Y. Gogotsi, Adv. Mater. Technol., 2019, 4, 1800639.

64 J. E. Bishop, C. D. Read, J. A. Smith, T. J. Routledge and D. G. Lidzey, Sci. Rep., 2020, 10, 6610.

65 T. Carey, S. Cacovich, G. Divitini, J. Ren, A. Mansouri, J. M. Kim, C. Wang, C. Ducati, R. Sordan and F. Torrisi, Nat. Commun., 2017, 8, 1202.

66 T.-C. Wu, A. De Luca, Q. Zhong, X. Zhu, O. Ogbeide, D.-S. Um, G. Hu, T. Albrow-Owen, F. Udrea and T. Hasan, npj 2D Mater. Appl., 2019, 3, 42.

67 Y. Wang, M. Mehrali, Y.-Z. Zhang, M. A. Timmerman, B. A. Boukamp, P.-Y. Xu and J. E. ten Elshof, Energy Storage Mater., 2021, 36, 318-325.

68 P. He, X. Tang, L. Chen, P. Xie, L. He, H. Zhou, D. Zhang and T. Fan, Adv. Funct. Mater., 2018, 28, 1801121.

69 Y. Shi, M. Osada, Y. Ebina and T. Sasaki, ACS Nano, 2020, 14, 15216-15226.

70 G. Hu, T. Albrow-Owen, X. Jin, A. Ali, Y. Hu, R. C. T. Howe, K. Shehzad, Z. Yang, X. Zhu, R. I. Woodward, T.-C. Wu, H. Jussila, J.-B. Wu, P. Peng, P.-H. Tan, Z. Sun, E. J. R. Kelleher, M. Zhang, Y. Xu and T. Hasan, Nat. Commun., 2017, 8, 278.

71 V. Bianchi, T. Carey, L. Viti, L. Li, E. H. Linfield, A. G. Davies, A. Tredicucci, D. Yoon, P. G. Karagiannidis, L. Lombardi, F. Tomarchio, A. C. Ferrari, F. Torrisi and M. S. Vitiello, Nat. Commun., 2017, 8, 15763.

72 E. B. Secor, P. L. Prabhumirashi, K. Puntambekar, M. L. Geier and M. C. Hersam, J. Phys. Chem. Lett., 2013, 4, 1347-1351.

73 K. Hassan, T. T. Tung, N. Stanley, P. L. Yap, F. Farivar, H. Rastin, M. J. Nine and D. Losic, Nanoscale, 2021, 13, 5356-5368.

74 A. Moridi, S. M. Hassani-Gangaraj, M. Guagliano and M. Dao, Surf. Eng., 2014, 30, 369-395.

75 G. Cummins and M. P. Y. Desmulliez, Circuit World, 2012, 38, 193-213.

76 X. Tian, J. Jin, S. Yuan, C. K. Chua, S. B. Tor and K. Zhou, Adv. Energy Mater., 2017, 7, 1700127.

77 L. Huang, Y. Huang, J. Liang, X. Wan and Y. Chen, Nano Res., 2011, 4, 675-684.

78 T. Carey, C. Jones, F. Le Moal, D. Deganello and F. Torrisi, ACS Appl. Mater. Interfaces, 2018, 10, 19948-19956.

79 S. Abdolhosseinzadeh, X. Jiang, H. Zhang, J. Qiu and C. (John) Zhang, Mater. Today, 2021, 48, 214-240.

80 S. Gilje, S. Han, M. Wang, K. L. Wang and R. B. Kaner, Nano Lett., 2007, 7, 3394-3398.

81 S. Das, B. Yang, G. Gu, P. C. Joshi, I. N. Ivanov, C. M. Rouleau, T. Aytug, D. B. Geohegan and K. Xiao, ACS Photonics, 2015, 2, 680-686.

82 J. E. Bishop, J. A. Smith, C. Greenland, V. Kumar, N. Vaenas, O. S. Game, T. J. Routledge, M. Wong-Stringer, C. Rodenburg and D. G. Lidzey, ACS Appl. Mater. Interfaces, 2018, 10, 39428-39434.
83 S. Ulična, B. Dou, D. H. Kim, K. Zhu, J. M. Walls, J. W. Bowers and M. F. A. M. Van Hest, ACS Appl. Energy Mater., 2018, 1, 1853-1857.

84 A. T. Barrows, A. J. Pearson, C. K. Kwak, A. D. F. Dunbar, A. R. Buckley and D. G. Lidzey, Energy Environ. Sci., 2014, 7, 2944-2950.

85 M. Park, W. Cho, G. Lee, S. C. Hong, M. Kim, J. Yoon, N. Ahn and M. Choi, Small, 2019, 15, 1804005.

86 A. G. Ricciardulli and P. W. M. Blom, Adv. Mater. Technol., 2020, 5, 1900972.

87 I. Burgués-Ceballos, M. Stella, P. Lacharmoise and E. Martínez-Ferrero, J. Mater. Chem. A, 2014, 2, 17711-17722.

88 A. G. Kelly, D. Finn, A. Harvey, T. Hallam and J. N. Coleman, Appl. Phys. Lett., 2016, 109, 23107.

89 S. Conti, L. Pimpolari, G. Calabrese, R. Worsley, S. Majee, D. K. Polyushkin, M. Paur, S. Pace, D. H. Keum, F. Fabbri, G. Iannaccone, M. Macucci, C. Coletti, T. Mueller, C. Casiraghi and G. Fiori, Nat. Commun., 2020, 11, 3566.

90 R. F. Hossain, I. G. Deaguero, T. Boland and A. B. Kaul, npj 2D Mater. Appl., 2017, 1, 28.

91 A. M. Alamri, S. Leung, M. Vaseem, A. Shamim and J. H. He, IEEE Trans. Electron Devices, 2019, 66, 2657-2661.

92 E. Brown, P. Yan, H. Tekik, A. Elangovan, J. Wang, D. Lin and J. Li, Mater. Des., 2019, 170, 107689.

93 R. Yang, J. Zhou, C. Yang, L. Qiu and H. Cheng, Adv. Mater. Technol., 2020, 5, 1901066.

94 D. Ponnamma, Y. Yin, N. Salim, J. Parameswaranpillai, S. Thomas and N. Hameed, Composites, Part B, 2021, 204, 108493.

95 R. Yang, J. Zhou, C. Yang, L. Qiu and H. Cheng, Adv. Mater. Technol., 2020, 5, 1901066.

96 K. Hassan, M. J. Nine, T. T. Tung, N. Stanley, P. L. Yap, H. Rastin, L. Yu and D. Losic, Nanoscale, 2020, 12, 19007-19042.

97 D. Ponnamma, Y. Yin, N. Salim, J. Parameswaranpillai, S. Thomas and N. Hameed, Composites, Part B, 2021, 204, 108493.

98 J. E. Smay, G. M. Gratson, R. F. Shepherd, J. Cesarano and J. A. Lewis, Adv. Mater., 2002, 14, 1279-1283.

99 C. Zhu and J. E. Smay, J. Rheol., 2011, 55, 655-672.

100 F. Guo, Y. Jiang, Z. Xu, Y. Xiao, B. Fang, Y. Liu, W. Gao, P. Zhao, H. Wang and C. Gao, Nat. Commun., 2018, 9, 881.

101 H. Guo, R. Lv and S. Bai, Nano Mater. Sci., 2019, 1, 101-115. 102 J. T. Muth, D. M. Vogt, R. L. Truby, Y. Mengüç, D. B. Kolesky, R. J. Wood and J. A. Lewis, Adv. Mater., 2014, 26, 6307-6312.

103 K. S. Kim, Y. Zhao, H. Jang, S. Y. Lee, J. M. Kim, K. S. Kim, J. H. Ahn, P. Kim, J. Y. Choi and B. H. Hong, Nature, 2009, 457, 706-710.

104 E. B. Secor, S. Lim, H. Zhang, C. D. Frisbie, L. F. Francis and M. C. Hersam, Adv. Mater., 2014, 26, 4533-4538.

105 Y. Cai, J. Shen, Z. Dai, X. Zang, Q. Dong, G. Guan, L. J. Li, W. Huang and X. Dong, Adv. Mater., 2017, 29, 1606411. 
106 C. Zhu, T. Y. J. Han, E. B. Duoss, A. M. Golobic, J. D. Kuntz, C. M. Spadaccini and M. A. Worsley, Nat. Commun., 2015, 6, 6962.

107 C. Zhu, T. Liu, F. Qian, T. Y. J. Han, E. B. Duoss, J. D. Kuntz, C. M. Spadaccini, M. A. Worsley and Y. Li, Nano Lett., 2016, 16, 3448-3456.
108 B. Yao, S. Chandrasekaran, J. Zhang, W. Xiao, F. Qian, C. Zhu, E. B. Duoss, C. M. Spadaccini, M. A. Worsley and Y. Li, Joule, 2019, 3, 459-470.

109 V. G. Rocha, E. García-Tuñón, C. Botas, F. Markoulidis, E. Feilden, E. D'Elia, N. Ni, M. Shaffer and E. Saiz, ACS Appl. Mater. Interfaces, 2017, 9, 37136-37145. 